\title{
Aortic root sizing for transcatheter aortic valve implantation using a shape model parameterisation
}

\author{
Bart Bosmans · Toon Huysmans · Patricia \\ Lopes · Eva Verhoelst - Tim Dezutter · Peter \\ de Jaegere · Jan Sijbers · Jos Vander Sloten . \\ Johan Bosmans
}

Received: date / Accepted: date

\begin{abstract}
During a transcatheter aortic valve implantation, an axisymmetric implant is placed in an irregularly shaped aortic root. Implanting an incorrect size can cause complications such as leakage of blood alongside or through the implant. The aim of this study was to construct a method that determines the optimal size of the implant based on the 3-dimensional shape of the aortic root. Based on the pre-interventional computed tomography scan of 89 patients, a statistical shape model of their aortic root was constructed. The weights associated with the principal components and the volume of calcification in the aortic valve were used as parameters in a classification algorithm. The classification algorithm was trained using the patients with no or mild leakage after their intervention. Subsequently, the algorithms were applied to the patients with moderate to severe leakage. Cross validation showed that a random forest
\end{abstract}

B. Bosmans $\cdot$ P. Lopes $\cdot$ J. Vander Sloten

KULeuven, Faculty of Engineering Science, Departement of Mechanical Engineering, Biomechanics Section, Celestijnenlaan 300C, 3001 Leuven, Belgium

Tel.: +3216396728

Fax: +3216396606

E-mail: Bart.Bosmans@kuleuven.be

B. Bosmans · P. Lopes · E. Verhoelst

Materialise N.V., Technologielaan 15, 3001 Leuven, Belgium

B. Bosmans $\cdot$ J. Bosmans

University of Antwerp, Faculty of Medicine and Health Sciences, Department of Translational Pathophysiological Research, Cardiovascular diseases, Universiteitsplein 1, 2610 Antwerp, Belgium

T. Huysmans $\cdot$ P. Lopes $\cdot$ J. Sijbers

University of Antwerp, iMinds-Vision Lab, Universiteitsplein 1, 2610 Antwerp, Belgium

T. Dezutter

UGent, IBiTech-bioMMeda, iMinds medical IT, Ghent University, Ghent, Belgium

T. Dezutter

FEops N.V., Technologiepark 3, 9052 Ghent, Belgium

P. de Jaegere

Erasmus Medical Center, Thoraxcenter, Departement of Cardiology, s-Gravendijkwal 230, 3015 CE Rotterdam, The Netherlands 


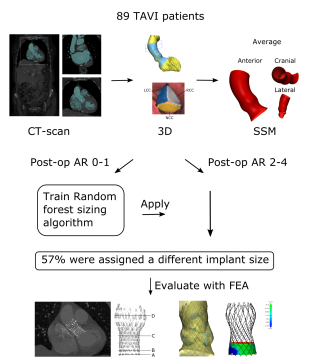

Fig. 1 The Graphical Abstract depicts a schematic overview of the methods and main results of this study

classifier assigned the same size in $65 \pm 7 \%$ of the training cases, while $57 \pm 8 \%$ of the patients with moderate to severe leakage were assigned a different size. This initial study showed that this semi-automatic method has the potential to correctly assign an implant size. Further research is required to assess whether the different size implants would improve the outcome of those patients.

Keywords Statistical shape modelling - Aortic root sizing - Transcatheter aortic valve implantation

\section{Introduction}

Aortic valve stenosis is the most commonly acquired valvular heart disease in the elderly. Despite advances in cardiac surgery and low mortality rates, up to one third of patients with symptomatic aortic valve stenosis are not considered for surgical valve replacement, often due to age, frailty or co-morbidities [4,34]. Transcatheter aortic valve implantation (TAVI) has been proven to be a reasonable alternative for the treatment of aortic valve stenosis in elderly (very) high-risk patients [20].

During the TAVI procedure an axisymmetric device is implanted in the patient's aortic root. In case of the CoreValve devices (Medtronic Inc., Minneapolis, MN, USA), four sizes are available, they have a $23 \mathrm{~mm}, 26 \mathrm{~mm}, 29 \mathrm{~mm}$, or $31 \mathrm{~mm}$ bottom cross-sectional diameter respectively. The CoreValve size range is used to treat patients with an annulus diameter between $18 \mathrm{~mm}$ and $29 \mathrm{~mm}$ [16]. The current planning procedure uses computed tomography (CT) images to size the annulus, the ring formed by the bottom of each valve leaflet. The annulus diameter can be calculated based on the perimeter, the cross-sectional surface area or the minimum and maximum diameter of the line delineating the aortic annulus $[10,15]$. However, the aortic root and the implants are 3-dimensional (3D) structures, the aortic root is rarely cylindrical and a suboptimal implant size can lead to complications such as aortic regurgitation (AR) $[13,19]$.

Determining the size of the implant based on the 3D shape of the aortic root might reduce complication and the observer dependency. Therefore, the goal of this research is to construct a method with which the implant size can be accurately estimated based on a parametric description of the $3 \mathrm{D}$ aortic root shape. 
Table 1 Clinical baseline characteristics

\begin{tabular}{lc}
\hline Variable & All patients $(\mathrm{N}=89)$ \\
\hline Female & $47(52.8 \%)$ \\
Age [years] & $80.3 \pm 7.2$ \\
Body mass index $\left[\mathrm{kg} / \mathrm{m}^{2}\right]$ & $27.7 \pm 4.5$ \\
EuroSCORE & $16.9 \pm 11.1$ \\
EuroSCORE2 & $5.6 \pm 4.1$ \\
STS & $5.4 \pm 3.3$ \\
Aortic valve area $\left[\mathrm{cm}^{2}\right]$ & $0.66 \pm 0.17$ \\
Mean gradient $[\mathrm{mmHg}]$ & $43.6 \pm 13.4$ \\
Peak gradient $[\mathrm{mmHg}]$ & $67.9 \pm 21.8$ \\
\hline
\end{tabular}

A statistical shape model (SSM) is a common method to generate a parametric description of a population of $3 \mathrm{D}$ shapes. In this type of model, each shape is described as a deviation from the average of the population along the principal components of variation $[11,36]$. SSMs have multiple applications in characterizing anatomical variability, for example, investigating the difference between the brain anatomy of healthy people versus schizophrenics [14] or Alzheimer patients [35]. SSMs are also used to investigate the shape variation in bone structures such as the human ear canal [26] and to reconstruct missing, malformed or fractured bone structures [29,37]. In addition, SSMs are used to model the whole body [25] or a part, such as the scalp [21] to provide a design space for clothing for example.

SSMs of the heart and vascular structures are described in literature. They are often used for the automatic segmentation of 3D cardiovascular images [17, 23, 24], and 4D images of the aorta [38] for example. Lekadir et al. (2016), Allen et al. (2016) and Pinto et al. (2016) used a statistical shape model (SSM) of the heart for the identification of myocardial infarction and to classify its severity $[2,22,28]$.

\section{Material and methods}

89 patients (table 1) from two centres, the University hospital in Antwerp and the Erasmus medical center in Rotterdam, received a contrast enhanced ECG-triggered, end diastolic CT scan prior to the TAVI procedure in which a CoreValve was implanted. The scan reconstruction resulted in a spatial resolution of $0.6 \mathrm{~mm}$ to $0.7 \mathrm{~mm}$ in-plane and $0.4 \mathrm{~mm}$ to $0.5 \mathrm{~mm}$ through-plane. All patients received an intravenous injection of $80 \mathrm{ml}$ of contrast agent at a flow rate of $4 \mathrm{ml} \mathrm{s}^{-1}$, followed by $30 \mathrm{ml}$ at $2.5 \mathrm{ml} \mathrm{s}^{-1}$. The minimum diameter, the maximum diameter, the diameter based on the perimeter and the diameter based on the surface area of the aortic annulus were taken into account to determine the size of the implant [30]. One patient received a $23 \mathrm{~mm}$ implant, 23 patients received a $26 \mathrm{~mm}$ implant, 50 patients received a $29 \mathrm{~mm}$ implant and 15 received a $31 \mathrm{~mm}$ implant. AR was graded immediately after the implantation on the procedural angiography, as described by Sellers et al. (1964) [31].

Starting from the pre-operative CT scans a parametric description of the 3D shape of the aortic root was generated. Subsequently, those parameters were used to perform a sizing of the aortic root for the implant size selection. Figure 2 depicts the steps in 


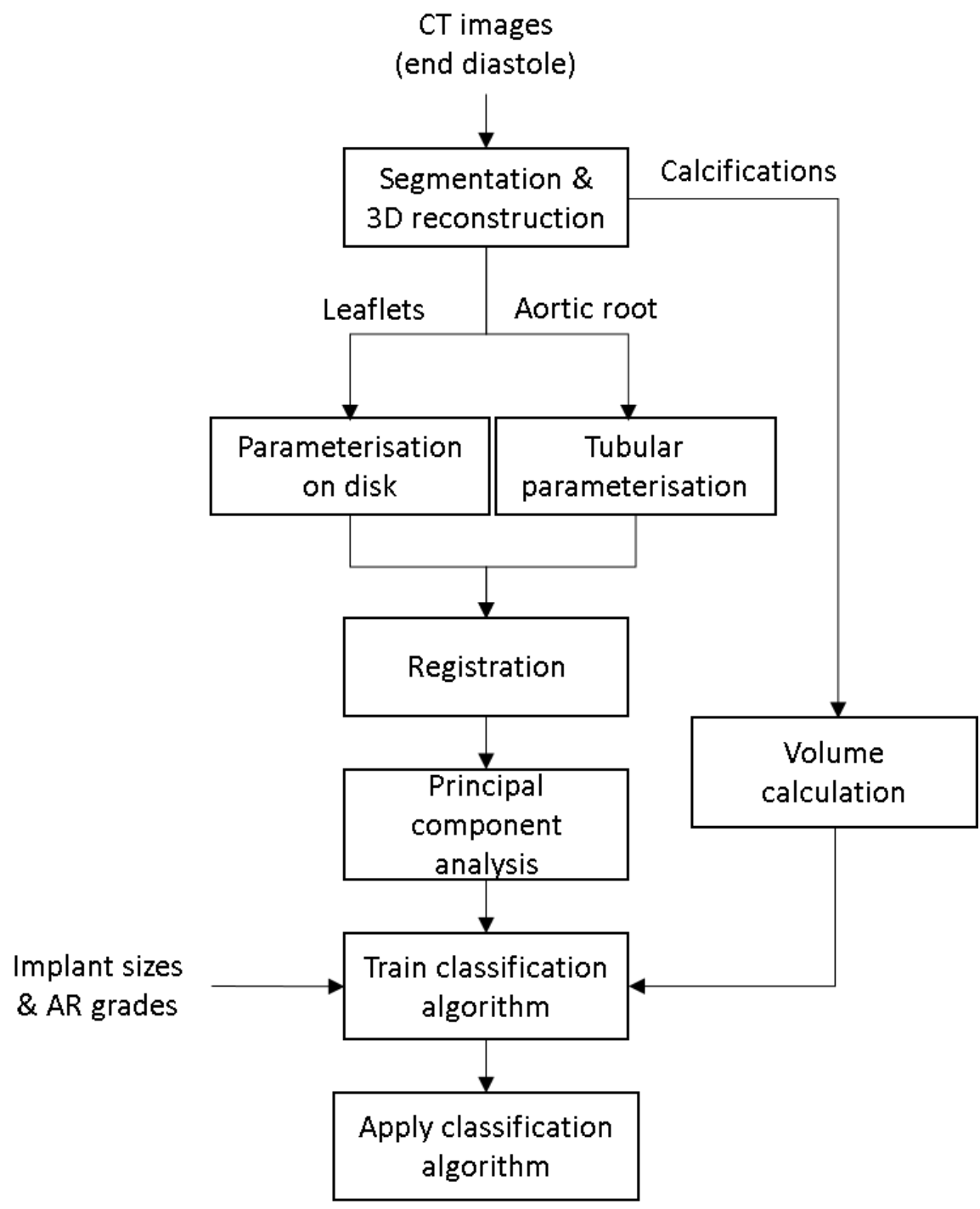

Fig. 2 flowchart of the data analysis process

the process from CT-images to the classification algorithm used for the automatic sizing of the aortic root.

\subsection{Image analysis}

Segmentation was performed on the pre-operative scans to extract the 3D shape of the aortic root using Mimics 16.0 (Materialise N.V., Leuven, Belgium). The left ventricle and aorta were extracted from the CT images using a threshold on the contrast agent 

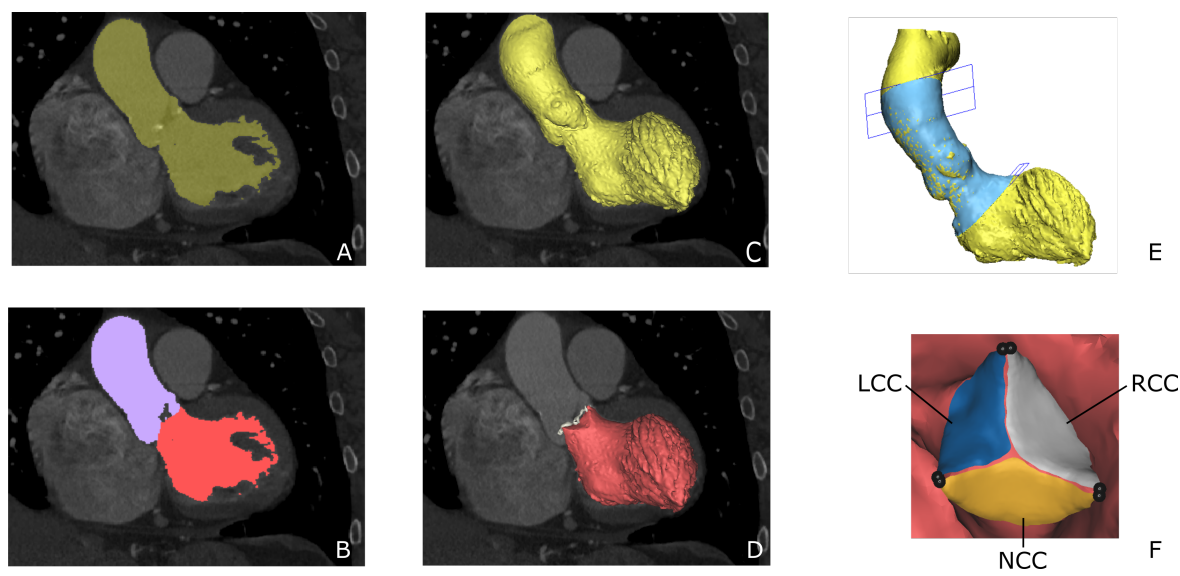

Fig. 3 (A) Coronal view of a computed tomography scan with the left ventricle and aorta blood pool coloured yellow. (B) Three dimensional (3D) reconstruction of the geometry. (C) The tubular aortic root model. (D) Split of the blood pool in aorta and left ventricle. (E) The 3D reconstruction of the left ventricle and aortic valve calcifications. $(\mathrm{F})$ The individual leaflets and commissure points used in the shape model generation (LCC: left coronary cusp, RCC: right coronary cusp and NCC: non-coronary cusp).

in the blood, as depicted in figure 3A. The left ventricle and aorta were separated from connected structures and each other using a graph cut algorithm [7] (figure 3B). $3 \mathrm{D}$ triangulated parts of the blood volume and the separate chambers were created using a marching cubes triangulation (figure 3C and 3D). Laplacian smoothing was performed to remove noise and small substructures. The aortic root was cut from the 3D part to create a tubular model using a plane perpendicular to the centerline at the level of the mitral valve and the aortic arch (3E). Three leaflets were created starting from the left ventricle model by smoothing and disconnecting the valve surface (3D). Finally, the calcifications were extracted using a threshold of 800 Hounsfield units, which is a consistently higher intensity compared to the contrast agent in the vast majority of patients. A region grow was applied to select the calcifications attached to the aortic valve, followed by a marching cubes triangulation to convert the pixels into a 3D model. Finally, the internal volume of the calcifications was computed.

\subsection{Generate the shape model}

The next step was to generate meshes of corresponding points from the population of surface models. A different method was used for the tubular part of the aortic root and the leaflets. The calculation of the corresponding points for tubular surfaces was described in detail in Huysmans et al. (2010) [18]. Briefly, first the tubular shape was mapped to an open-ended cylinder. Next, the shapes were aligned using their principal axes, then the alignment of the parameterisations was determined by minimizing the description length of the SSM [12]. Finally, both the spatial alignment and the parameterisations were optimised simultaneously with respect to the minimum description length. 


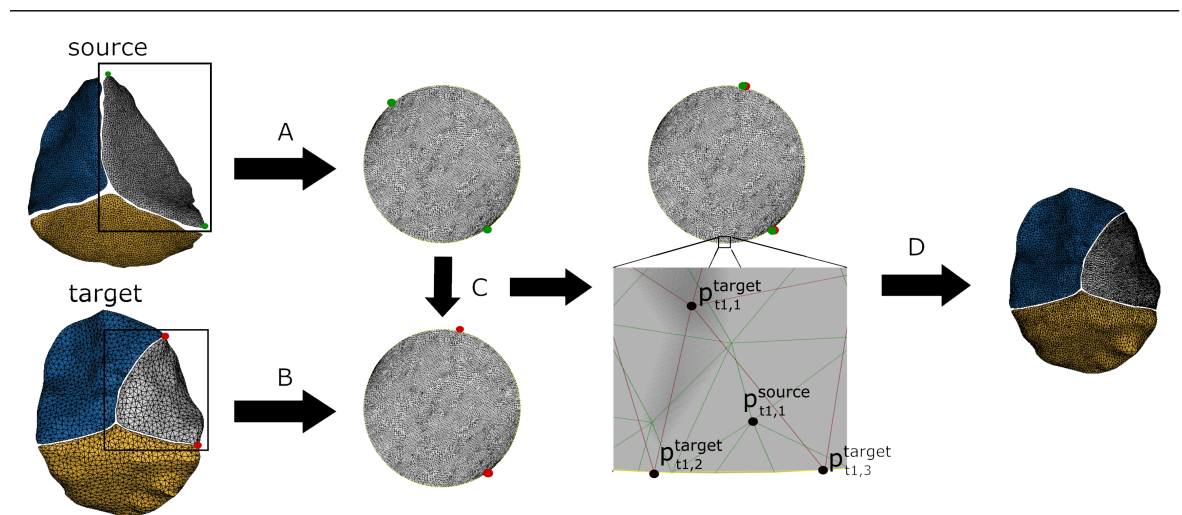

1
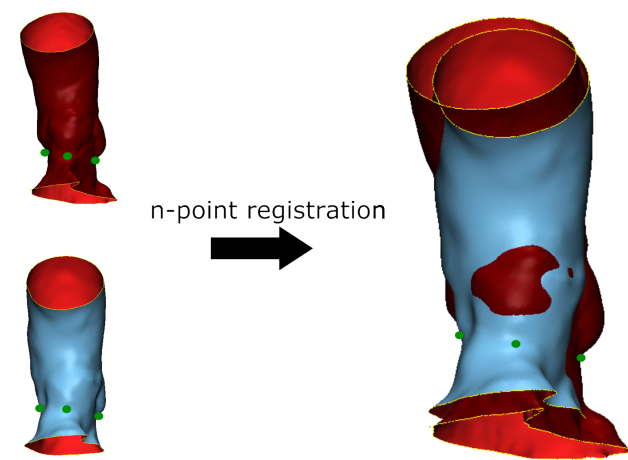

2

Fig. 4 part 1: (A) parameterisations of the master leaflet on a disk with diameter 1. (B) parameterisations of a sample leaflet on a disk. (C) Registration of the master parameterisations on the sample using the commissure points. (D) Transformation of the corresponding points of the master parameterisations to the sample leaflet. part 2: The registration of two 3D models of two different patients based on the annulus plane

The correspondence in the leaflets was determined using a mapping of each leaflet on a disk with diameter one. The mapping was determined by representing each point on the surface as a linear combination of its neighbours. This resulted in a system of linear equations that has a unique solution starting from the points on the boundary. These boundary points were intialised on the disk perimeter at a point to point distance equivalent to the relative distance along the boundary of the leaflet. The disks were aligned along the section of the boundary between the two leaflet commissures which denote the attachment to the aortic wall. Next, a Laplacian smoothing was performed on the first instance of each of the three leaflets, in order to generate a more uniform mesh to describe all the leaflet meshes. The smoothing substitutes each point with the average of its neighbours, constructing the master parameterisations. Subsequently, the points of this master parameterisations were transformed to each patients leaflets. The transformation is determined by the location of the points of the master inside the triangles of the parameterised target leaflet (figure 4 part 1). 
Next, the SSM is built, first the spatial registration derived from the aortic root parameterisations was applied to the leaflets to position them correctly inside each shape. Next the shapes of different patients are registered onto each other. The registration was performed based on the aortic annulus plane in order to minimize the registration error with respect to the leaflets, and the aortic annulus since that was considered the region of most interest for sizing (figure 4 part 2). The shapes are represented as a vector $x_{i}=\left[x_{i, 1}, y_{i, 1}, z_{i, 1}, \ldots, x_{i, n}, y_{i, n}, z_{i, n}\right]$ and put into a matrix as columns. After subtracting the mean shape $\bar{x}$ of each column, matrix A contained the deviation from the mean shape.

$$
A=\left[x_{1}^{T}-\bar{x}^{T} \ldots x_{m}^{T}-\bar{x}^{T}\right]
$$

Next, the principal components were computed by performing a singular value decomposition. Each aortic root could then be represented as a sum of the mean and the weighted principal components:

$$
x=\bar{x}+U b
$$

where $x$ is a new shape, $U$ a matrix with the principal components as columns and $b$ a column vector of the weights of the principal components.

The quality of the SSM was evaluation using the compactness, generalisation ability and specificity $[18,32]$. The compactness was calculated as the percentage of the total variance present in the first $\mathrm{n}$ principal components:

$$
C(n)=\frac{\sum_{i=1}^{n} \lambda_{i}}{\sum_{i=1}^{m} \lambda_{i}}
$$

where $\lambda_{i}$ is the variance of the $\mathrm{i}^{\text {th }}$ principal component and $\mathrm{m}$ the total number of principal components. The generalisation ability is a measure for the ability of the SSM to approximate a shape which is not in the model and was calculated using a leave-one-out experiment. The specificity measures how close random samples, generated by the model are to the shapes in the population.

\subsection{Size classification}

The principal component analysis aggregates the total shape variation in a limited number of independent principal components. Therefore, a good approximation of a shape can be obtained using only those first principal components. In the aortic root model $95 \%$ of all variation was described by the first 20 principal components.

Based on the assumption that the patients with post-operative AR grade 0 - 1 received the correct implant size, the patients were divided in two groups: the first group had AR grade $0-1$, the second group had an AR grade $\geq 2$. The first group was used to train two different classification algorithms, a generalised linear model, since the parameters are the linear independent modes of variation of the SSM, and a random forest classifier which does not impose a model on the data [27].

The weights of the first 20 principal components and the aortic valve calcification volume were used as parameters to describe each patient. In a first step, these 
parameters were ranked using an analysis of variance to determine which parameters discriminated most between the size groups. Next, both classification algorithms were trained incrementally including additional parameters.

Two parameters describe the random forest used in the classifier: first, the number of trees determines the amount of independent classifiers that will be part of the forest from which the majority answer is chosen. Due to the limited size of the training set and the small number of features, the number of random subsets from which to build each tree was small. Therefore, a small number of trees was chosen as starting point (10). Second, the depth of the trees determines the amount of splits available to classify a sample. Since, the training set and the number of parameters is limited in size, and there are only three possible outcome sizes, the minimum depth of 5 was taken as start point. During the investigation of the classification algorithm, the amount of trees and the depth was varied both increasing and decreasing. The values which gave the most consistent and best classification with the least amount of computing time were selected.

Each instance of the models was cross-validated by dividing the training set in 8 subgroups, then the model was trained excluding one group. The sizes of this remaining groups of patients were fitted and compared to their true size. When performed for each subgroup this resulted in a mean accuracy score and a standard error.

The best scoring classifier was applied to the patients with an AR grade $\geq 2$. A chi-squared test was used to test whether the classification of the patients with an AR grade $\geq 2$ was significantly different from the training set.

The analysis was performed in python 3.5 using the scikit-learn 0.17 machine learning module [27].

\subsection{Verification through implantation simulation}

Of the 36 patients with $\mathrm{AR}$ grade $\geq 2$ included in this study, a small subset also received a post-operative $\mathrm{CT}$ scan (figure $5 \mathrm{~L}$ ), two of those patients were assigned a different size implant by the random forest classifier. The implantation of both the implanted device and the newly assigned device was simulated in both patients using the method described in [6].

Briefly, a finite element model of the stent and the aortic root of the patient was made based on a micro-CT of the stent and the pre-operative CT of the patient. First, the loading of the stent on the catheter was simulated. Next, the stent was introduced in the aortic root model and released. Subsequently, the result of this simulation was used to calculate paths on the distance map between the sealing skirt of the implant and the simulated anatomy as an estimate of the AR after implantation.

First, the implantation of the given implant size is simulated and compared to the post-op CT in order to assess the accuracy of the simulation for both patients. At 4 axial cross sections (figure 5R), the centre of the stent struts was used to draw a line and calculate its perimeter, to quantify the stent dimensions of both the simulated stent and the stent from the post-operative CT. Next, the implantation of the different size implants was simulated and the resulting AR was estimated. 

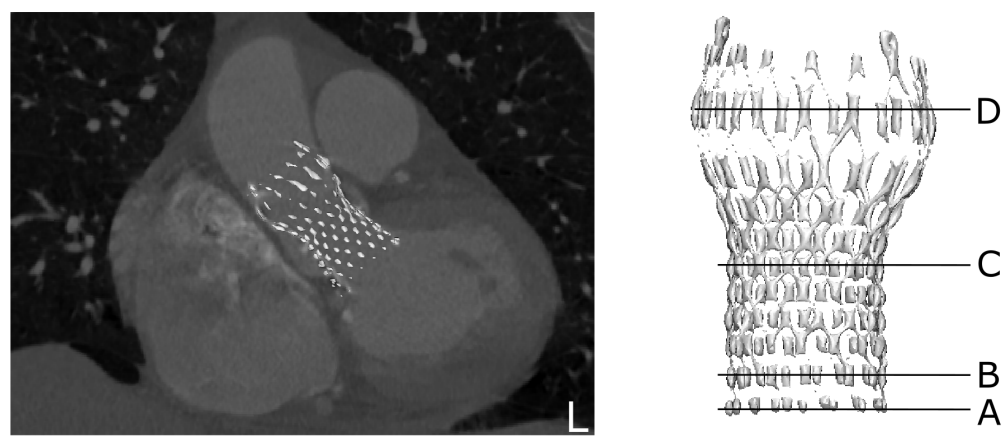

$\mathrm{R}$

Fig. 5 (L) A coronal view of the post-operative computed tomography scan of patient 5 with the segmented stent. (R) An overview of the locations of the cross section diameter measurements.

Table 2 Implant sizes distribution in the patient population

\begin{tabular}{ccc}
\hline CoreValve size $[\mathrm{mm}]$ & AR grade $0-1(\mathrm{n}=48)$ & AR grade $\geq 2(\mathrm{n}=36)$ \\
\hline 26 & $12(25 \%)$ & $9(24 \%)$ \\
29 & $30(62.5 \%)$ & $18(49 \%)$ \\
31 & $6(12.5 \%)$ & $9(24 \%)$ \\
\hline
\end{tabular}

\section{Results}

Of the 89 patients, 2 parameterisations failed, the algorithm was unable to find the corresponding points, 2 did not receive an angiographic AR evaluation immediately after implantation, and only one patient received a $23 \mathrm{~mm}$ implant. The remaining 84 patients were included in the analysis, $48(57 \%)$ patients had an AR grade 0 or 1, $37(43 \%)$ had an AR grade $\geq 2$. Table 2 gives an overview of the distribution of the implant sizes in the population divided according to the severity of AR. The median aortic valve calcification volume was $195.8 \mathrm{~mm}^{3}$, with the $25 \%$ quartile at $83.3 \mathrm{~mm}^{3}$ and the $75 \%$ quartile at $335.7 \mathrm{~mm}^{3}$.

\subsection{Statistical shape model}

Figure 6 depicts the first 3 principal components. The first principal component contains a general size variation. The second principal component contains curvature variations and changes in the angle of the aorta with respect to the annulus plane. The third component contains diameter variations and additional curvature variation. Figure 7 the compactness, and figure 8 depicts the generalisation ability and the specificity of the SSM. The compactness analysis shows that $48 \%$ of all shape variation is combined in the first two principal components, $87 \%$ of the variation is in the first 10 principal components and $95 \%$ of the variation is in the 20 first principal components. The generalisation ability shows that using the first 20 principal components, 
-3 std

PC1
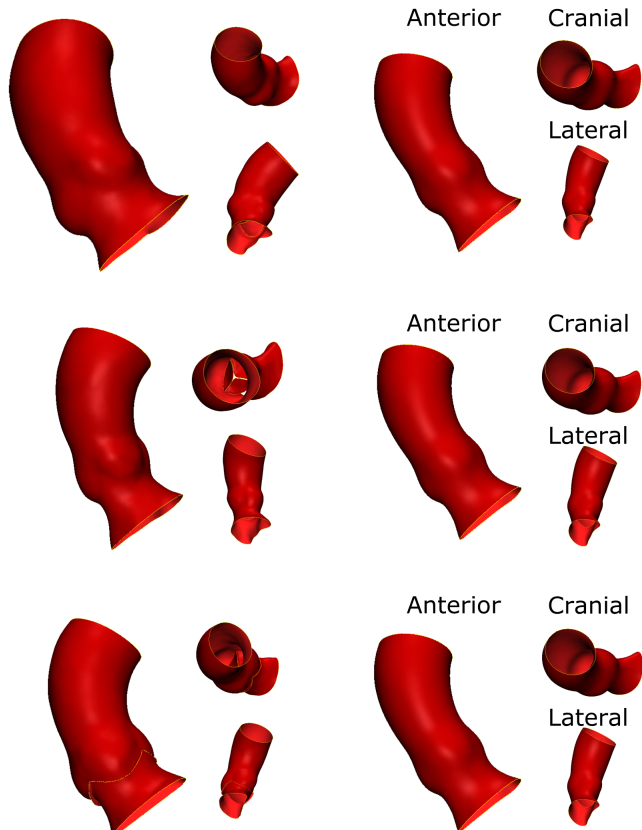

PC2

PC3

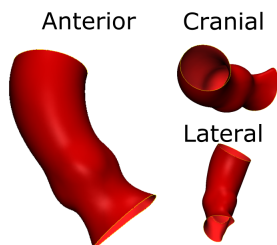

Average

$+3 \mathrm{std}$
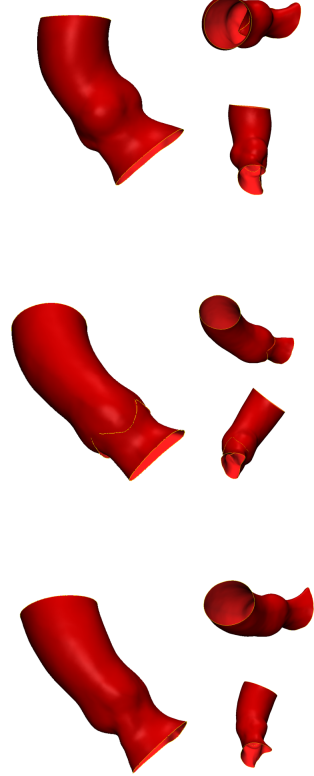

Fig. 6 A visualisation of the first 3 principal components (PC) of the statistical shape model, the anterior, cranial and lateral view of the average \pm 3 standard deviations are shown.

a new shape can be approximated with a mean absolute distance of $1 \mathrm{~mm}$. Finally, as described by the specificity analysis (figure 8), randomly generated shapes are at a mean absolute distance of approximately $3 \mathrm{~mm}$ from the closest shape in the training population.

\subsection{The sizing algorithm}

Figure 9 depicts the result of the parameter selection analysis, both classification algorithms perform best using the $20 \%$ most discriminating features. These features are principal component $1,10,7$ and 2 respectively. Figure 10 depicts principal components 7 and 10. Both principal component 7 and 10 contain local diameter variations at the level of the aortic root. Principal component 7 also shows variations of the length of the aortic root model. Principal components 1 and 2 are shown in figure 6 .

The linear model assigns $69 \pm 7 \%$ of the included patients the same size as they had implanted, the random forest classifier assigns the same size to $96 \pm 3 \%$ when applying the classifier to the test data. Table 3 gives an overview of the cross-validation results of the random forest model. The F-score for a $26 \mathrm{~mm}$ implant is 0.56 , and the F-score for a $29 \mathrm{~mm}$ implant is 0.76 . 


\section{Compactness}

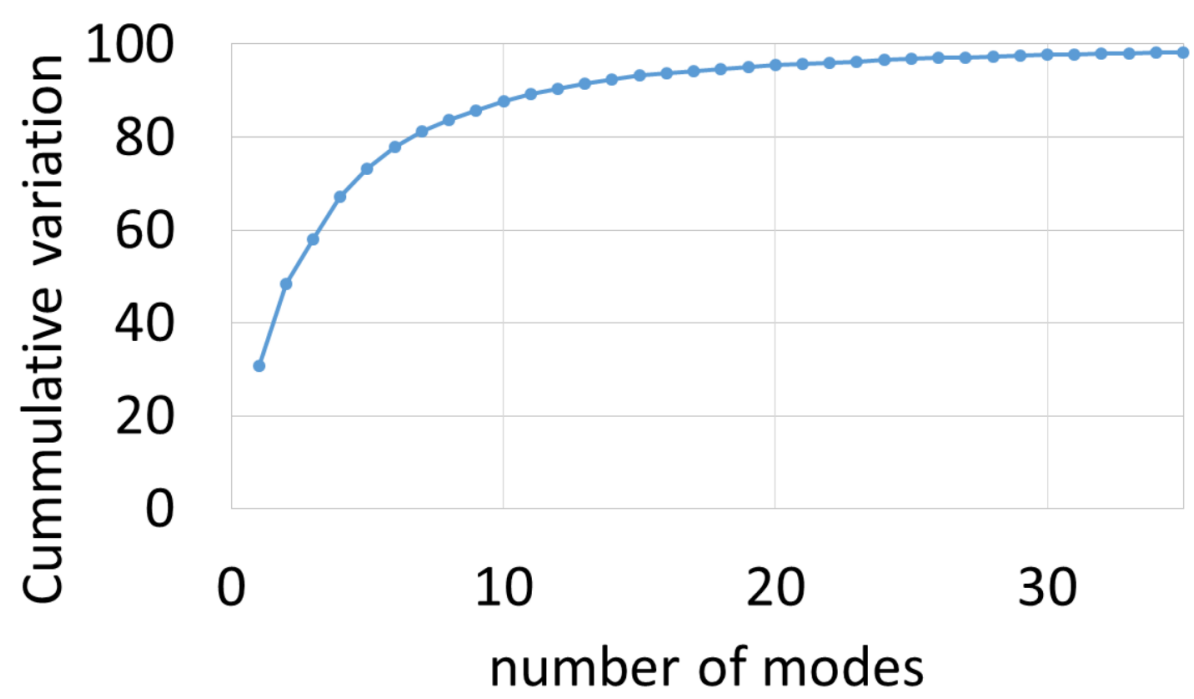

Fig. 7 The compactness of the statistical shape model of the aortic root
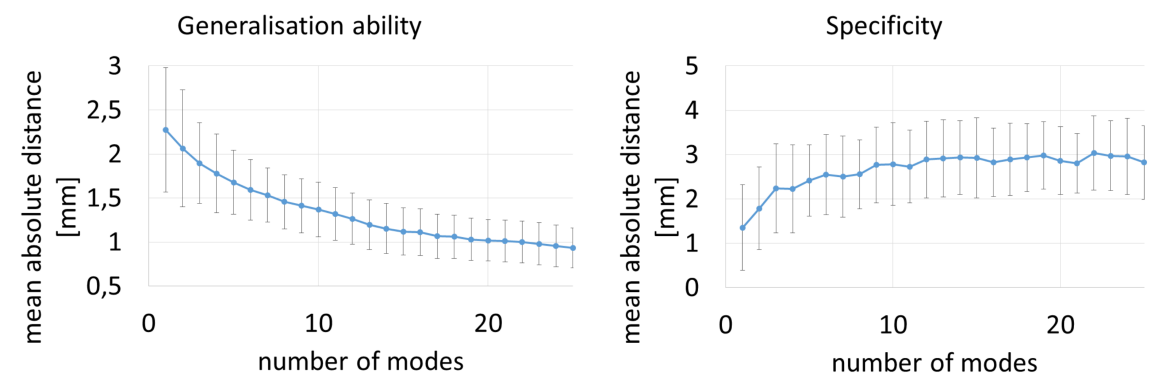

Fig. 8 The statistical shape model evaluation: left, the generalisation ability \pm 1 standard deviation and right the specificity \pm 1 standard deviation.

Table 3 Cross-validation of the training set: confusion matrix of the random forest classifier

\begin{tabular}{ccccc} 
& & \multicolumn{3}{c}{ predicted } \\
& & 26 & 29 & 31 \\
\multirow{3}{*}{ actual } & 26 & 7 & 5 & 0 \\
& 29 & 4 & 24 & 2 \\
& 31 & 2 & 4 & 0
\end{tabular}



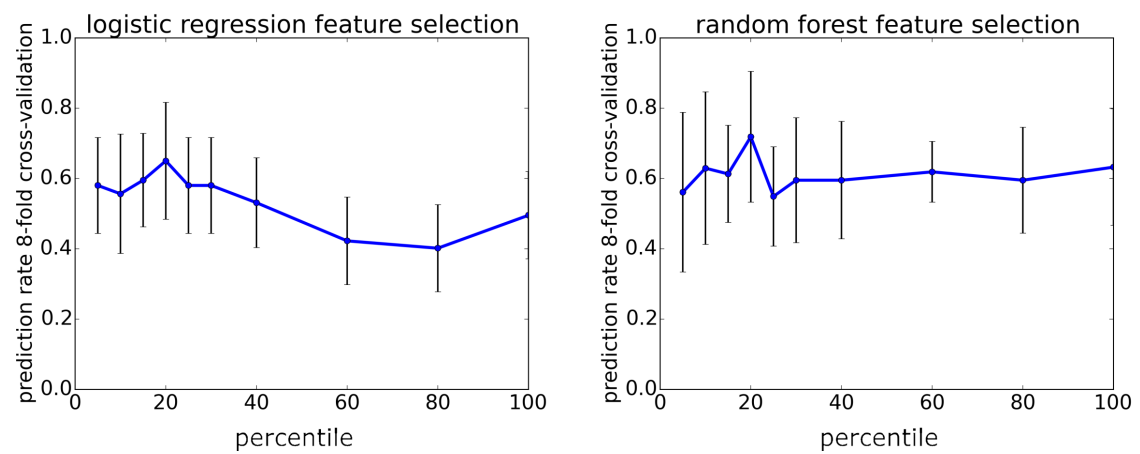

Fig. 9 the result of the 8-fold cross-valdition and standard error for both classification algorithms as a function of the percentile of parameters included in the model.

$$
-3 \text { std }
$$

Average

$+3 \mathrm{std}$
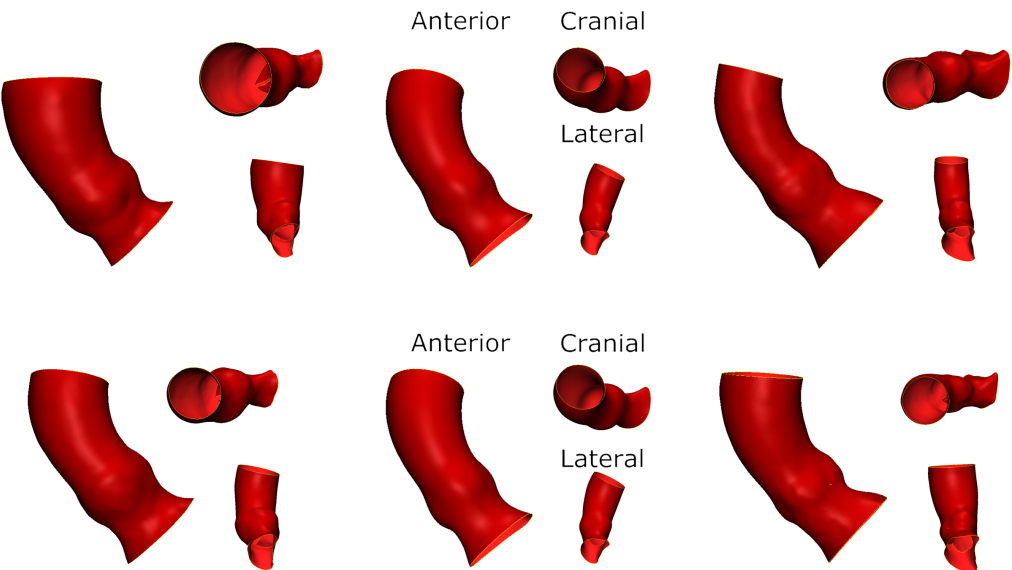

Fig. 10 A visualisation of principal components (PC) 7 and 10 of the statistical shape model, the anterior, cranial and lateral view of the average \pm 3 standard deviations are show.

Table 4 contains the results of the random forest classification algorithms on the patients with $\mathrm{AR} \geq 2$. The average difference between the predicted size and the implanted size is $-0.14 \pm 2.04 \mathrm{~mm}$ for the random forest classifier. The random forest classifier assigned the same size to 16 out of 36 patients with $A R \geq 2,10$ patients got one size larger, 1 got two sizes larger and 9 got one size smaller.

Overall, the random forest classifier assigned the same size in $65 \pm 7 \%$ of the patients with AR grade 0 or 1 , and in $43 \pm 8$ of the patients with AR grade $\geq 2$. Due to the limited size of the included patient population, the classification of the patients with $A R \geq 2$ is not significantly different from the cross-validation of the training set $(p=0.28)$. Even, specifically for the $29 \mathrm{~mm}$ size subgroups there is no statistical significant difference $(\mathrm{p}=0.33)$. 
Table 4 Implant sizes assigned to patients with $\mathrm{AR} \geq 2$

\begin{tabular}{ccccc} 
& & \multicolumn{3}{c}{ predicted } \\
& & 26 & 29 & 31 \\
\multirow{3}{*}{ received } & 26 & 2 & 6 & 0 \\
& 29 & 4 & 11 & 3 \\
& 31 & 2 & 7 & 2
\end{tabular}

Table 5 The perimeters of the simulated stents that were implanted in the patients and the difference with the post-op and the alternative stent size

\begin{tabular}{llcccc}
\hline & & Section A & Section B & Section C & Section D \\
\hline \multirow{2}{*}{ Patient A } & perimeter [mm] & 68.7 & 68.4 & 63.7 & 81.9 \\
$26 \mathrm{~mm}$ & $\Delta$ post-op [mm] & 1.0 & 0.6 & 1.7 & 1.6 \\
& $\Delta$ 29 mm [mm] & 2.8 & 5.6 & 5.2 & -3.2 \\
Patient B & perimeter [mm] & 74.1 & 76.4 & 68.4 & 96.9 \\
$29 \mathrm{~mm}$ & $\Delta$ post-op [mm] & 10.1 & 5.9 & -1.3 & 0.4 \\
& $\Delta$ 26 mm [mm] & -3.2 & -6.3 & -5.7 & -2.1 \\
\hline
\end{tabular}

Table 6 The AR estimation with the two different implant sizes

\begin{tabular}{lccc}
\hline & Max-flow [mm] & $\begin{array}{c}\text { Post-op Angiographic } \\
\text { AR grade [0 - 4] }\end{array}$ \\
\hline \multirow{2}{*}{ Patient A } & $26 \mathrm{~mm}$ & 177.3 & 3 \\
& $29 \mathrm{~mm}$ & 7.0 & 3 \\
\multirow{2}{*}{ Patient B } & $26 \mathrm{~mm}$ & 161.6 & \\
& $29 \mathrm{~mm}$ & 51.2 & \\
\hline
\end{tabular}

3.3 Verification through implantation simulation

Patient A had a $26 \mathrm{~mm}$ CoreValve implanted and was assigned a $29 \mathrm{~mm}$ implant by the algorithm with a probability of $90 \%$. Patient B had a $29 \mathrm{~mm}$ implant and was assigned a $26 \mathrm{~mm}$ CoreValve with a $71 \%$ probability by the random forest classifier.

Table 5 shows the measurements performed on the simulated stents. The comparison with the post-operative data shows that the simulation of patient $\mathrm{A}$ is very close to the measurements on the post-operative scan, while in patient $\mathrm{B}$ the simulation underestimates the perimeter at the bottom of the stent. Comparing the proposed size with the implanted size the perimeter change is similar in magnitude, an increase and decrease respectively of the perimeter with $3 \mathrm{~mm}$ to $6 \mathrm{~mm}$.

Table 6 contains the AR estimation based on the simulated implantations. In patient A, the $29 \mathrm{~mm}$ stent results in a significant reduction of the estimated flow. In Patient B, a threefold increase of the flow is estimated with the proposed $26 \mathrm{~mm}$ stent. Upon further investigation the calculated flow for the $29 \mathrm{~mm}$ stent, implanted in patient B, is low for AR grade 3, and the simulation of patient B was less accurate with respect to the post-operative CT. 


\section{Discussion}

The goal of this research was to construct a method to perform the sizing of the aortic root for implant selection based on its 3D shape. The method described in this study used the weights associated with the principal components of a SSM as a parametric description of the 3D aortic root shape of each patient. The weights and the volume of the calcifications were used as parameters in two classification algorithms: a generalised linear model and a random forest classifier. The classification algorithms were trained using the patients who did not suffer from AR (grade 0 ) or only suffered from mild AR (grade 1) after implantation of the device, assuming that those patients received the optimal implant size.

The random forest classification algorithm performed best during the training, assigning the correct implant to $96 \pm 3 \%$ of the patients in the training cohort. The 8 -fold cross-validation on the same training cohort resulted in $65 \pm 7 \%$ correctly assigned implant sizes. The cross-validation showed that the robustness of the classification algorithm was limited by the number of patients in the implant size subgroups. For example, the subgroup of patients with a $31 \mathrm{~mm}$ implant did not contain enough patients to train the algorithm when some were left out as part of the cross validation experiments. The higher prevalence of $29 \mathrm{~mm}$ implant patients in the training set resulted in a better F-score for those patients.

Applying the trained algorithm to the patients with moderate to severe AR showed $43 \pm 8$ of patients being assigned the same size as was implanted. Compared to the cross-validation result of the training set, this result implied that there was a difference between the patients with AR grade 0 or 1 and the patients with AR grade $\geq 2$. The patients could potentially benefit from the alternatively assigned implant size. However, the difference was not statistically significant.

The implantation of the stent assigned by the algorithm was simulated in two patients in order to get some insight in the benefit of the alternative stent size for the patient. The improvement of patient A is an encouraging result. However, patient B shows that additional research and data are needed to validate the automatic sizing algorithm. The evaluation of the implant performance based on a simulation is limited by the accuracy of the simulation for each patient. Therefore, two patients who had a post-op CT were used for this test.

In order to determine the size of a new patient's valve, the shape model needs to be fitted on the aortic root of that patient. The fit results in the weights associated with the principal components describing the new patient, these weights are then used in the classification algorithm. Two approaches can be devised to perform the fitting, either based on the displacement of corresponding points, therefore, the surface model of the aortic root needs to be corresponded with the SSM, or based on a fit of the surface using a non-rigid registration algorithm.

Only AR was investigated in this study, other complication, potentially linked with sizing, such as conduction problems were not considered.

The study had a number of limitation: first, only images of the diastolic phase were available, CT images of the systolic phase would show the annulus at its largest which are suggested to be better suited for the sizing and subsequent implant selection [3]. 
The accuracy of the model representing the anatomical variation depends on the accuracy of the segmentation. The segmentation accuracy of bone tissue was previously investigated [8]. The segmentation of soft tissue is more difficult to validate. However, a comparison between manual segmentation by experts and the segmentation performed by the algorithm showed a similar variation between the algorithm and the manual segmentation as the variation between two manual segmentations. Further investigation comparing images from different modalities could be used to validate the segmentation of soft tissue.

Since there are no landmarks that mark the start of the aortic root, a manual cut is used to determine the upper boundary of the model. It is likely that non anatomical length variations are part of the statistical shape model which could overshadow the true anatomical variation of the length.

The distribution and shape of the calcifications could influence the sealing of the implant, therefore it would be interesting to include both in the sizing research. However, including the calcifications as part of the shape model is not possible with the technique used in this study, due to the irregular nature of the their shape and position. A previous investigation in the same dataset could not find clear evidence of the significance of calcification distribution described by ratios between the leaflets [5].

In addition, the model could be extended to include the aortic arch and part of the descending aorta as an indication of the complexity of the approach to the aortic root. A tortuous approach could adversely influence correct placement of the implant leading to a higher risk of post-operative AR grade.

The principal components describing the shape variation that were used in the classification algorithms had no straightforward physical interpretation. A potential method to make the model more intuitive is to associate interpretable morphological measures with the principal components through correlation as described by Bruse et al. (2015) [9].

The inclusion of the leaflets caused some artifacts in the SSM, as can be seen in principal component 3 in figure 6 where the leaflets protrude through the aortic wall in the -3 standard deviation extreme. This can be due to the separate registration and parameterisation of the leaflets and the aortic root. Therefore, there is no physical connection in the model, nor correspondence, between the leaflet border and the aortic root. This can only be solved by constructing the correspondences of the leaflets and the wall as a whole. A registration based approach using a complete model of the aortic root, including the leaflets, as a master parameterisation could be a solution [23].

Another limitation that can cause the anatomically incorrect shapes, is the use of principal component analysis to perform the dimensionality reduction. It imposes linearity on the shape variation while the population of aortic roots, including the leaflets might contain substantial non-linear shape variation. Non-linear dimensionality reduction algorithms could improve the shape model $[1,24,33]$.

Finally, the study was limited by the size of the data set therefore, the statistical analysis showed no significant difference between the cross-validation of the training set and the sizing of the patients with $\mathrm{AR}$ grade $\geq 2$. Also, the feature selection started 
from a rather large number of features with respect to the amount of data points. Therefore, the results could alter when more patients would be included in the study.

\section{Conclusion}

The proposed method provides a semi-automatically tool to assign an implant size based on a pre-operative CT scan of the aortic root. It could provide the clinical team with additional information during the planning of the intervention. The results of this study suggest that when the algorithm assigns a larger size implant than the manufacturing guidelines, choosing the larger size could reduce the occurrence of AR.

Additional data of patients in the less prevalent implant sizes is needed to make the algorithm more robust. In addition, further research is required to include the fitting of the shape model to the work flow, and additional simulations need to be performed in order to further assess the effectiveness of the alternative implant size assigned to patients with moderate to severe AR.

\section{Conflicts of interest statement}

Prof. Dr. Johan Bosmans and Prof. Dr. Peter de Jaegere are part-time clinical proctor for Medtronic. Prof. Dr. ir. Jos Vander Sloten is a member of the Board of Directors of Materialise N.V. and a shareholder. The remaining authors have no conflicts of interest to declare.

Acknowledgements This work was supported in part by a PhD grant (120198) from the agency for innovation through science and technology (IWT) of the Flemish government.

\section{References}

1. Alizadeh Sani, Z., Shalbaf, A., Behnam, H., Shalbaf, R.: Automatic computation of left ventricular volume changes over a cardiac cycle from echocardiography images by nonlinear dimensionality reduction. Journal of Digital Imaging 28(1), 91-98 (2015)

2. Allen, J., Zacur, E., Dall'Armellina, E., Lamata, P., Grau, V.: Myocardial Infarction Detection from Left Ventricular Shapes Using a Random Forest, pp. 180-189. Springer International Publishing (2016)

3. Blanke, P., Schoepf, U.J., Leipsic, J.A.: Ct in transcatheter aortic valve replacement. Radiology 269(3), 650-669 (2013)

4. Bose, A.K., Aitchison, J.D., Dark, J.H.: Aortic valve replacement in octogenarians. Journal of cardiothoracic surgery 2, 33-5 (2007)

5. Bosmans, B., Collas, V., Verhoelst, E., Paelinck, B., Vander Sloten, J., Bosmans, J.: Morphological characteristics and calcification of the native aortic valve and the relation to significant aortic regurgitation post CoreValve TAVI. Journal of Heart Valve Disease (2016)

6. Bosmans, B., Famaey, N., Verhoelst, E., Bosmans, J., Vander Sloten, J.: A validated methodology for patient specific computational modeling of self-expandable transcatheter aortic valve implantation. Journal of Biomechanics 49(13), 2824-2830 (2016)

7. Boykov, Y., Kolmogorov, V.: An experimental comparison of min-cut/max-flow algorithms for energy minimization in vision. IEEE transactions on pattern analysis and machine intelligence 26(9), 112437 (2004) 
8. den Broeck, J.V., Vereecke, E., Wirix-Speetjens, R., Sloten, J.V.: Segmentation accuracy of long bones. Medical engineering \& physics 36(7), 949-953 (2014)

9. Bruse, J.L., Mcleod, K., Biglino, G., Ntsinjana, H.N., Capelli, C., Hsia, T.y., Sermesant, M., Pennec, X., Taylor, A.M., Schievano, S., Hearts, C.: A statistical shape modelling framework to extract 3D shape biomarkers from medical imaging data : assessing arch morphology of repaired coarctation of the aorta. BMC Medical Imaging 16(1), 40 (2016)

10. Buzzatti, N., Maisano, F., Latib, A., Cioni, M., Taramasso, M., Mussardo, M., Colombo, A., Alfieri, O.: Computed tomography-based evaluation of aortic annulus, prosthesis size and impact on early residual aortic regurgitation after transcatheter aortic valve implantation. European journal of cardiothoracic surgery : official journal of the European Association for Cardio-thoracic Surgery 43(1), 43-51 (2013)

11. Cootes, T.F., Hill, A., Taylor, C.J., Hastam, J.: Use of active shape models for locating structures in medical images. Image and Vision Computing 12(6), 355-365 (1994)

12. Davies, R.H., Twining, C.J., Cootes, T.F., Waterton, J.C., Taylor, C.J.: A minimum description length approach to statistical shape modeling. IEEE Transactions on Medical Imaging 21(5), 525-537 (2002)

13. Détaint, D., Lepage, L., Himbert, D., Brochet, E., Messika-Zeitoun, D., Iung, B., Vahanian, A.: Determinants of significant paravalvular regurgitation after transcatheter aortic valve: implantation impact of device and annulus discongruence. JACC. Cardiovascular interventions 2(9), 821-7 (2009)

14. Ferrarini, L., Palm, W.M., Olofsen, H., Van Der Landen, R., Van Buchem, M.A., Reiber, J.H.C., Admiraal-Behloul, F.: Ventricular shape biomarkers for Alzheimer's disease in clinical MR images. Magnetic Resonance in Medicine 59(2), 260-267 (2008)

15. Hayashida, K., Bouvier, E., Lefèvre, T., Hovasse, T., Morice, M.C., Chevalier, B., Romano, M., Garot, P., Mylotte, D., Farge, A., Donzeau-Gouge, P., Cormier, B.: Impact of CT-guided valve sizing on postprocedural aortic regurgitation in transcatheter aortic valve implantation. EuroIntervention : journal of EuroPCR in collaboration with the Working Group on Interventional Cardiology of the European Society of Cardiology 8(5), 546-55 (2012)

16. Holmes, D.R., Mack, M.J., Kaul, S., Agnihotri, A., Alexander, K.P., Bailey, S.R., Calhoon, J.H., Carabello, B.A., Desai, M.Y., Edwards, F.H., Francis, G.S., Gardner, T.J., Kappetein, A.P., Linderbaum, J.A., Mukherjee, C., Mukherjee, D., Otto, C.M., Ruiz, C.E., Sacco, R.L., Smith, D., Thomas, J.D.: 2012 ACCF/AATS/SCAI/STS Expert Consensus Document on Transcatheter Aortic Valve Replacement. Journal of the American College of Cardiology 59(13), 1200-1254 (2012)

17. Hoogendoorn, C., Duchateau, N., Sanchez-Quintana, D., Whitmarsh, T., Sukno, F.M., Craene, M.D., Lekadir, K., Frangi, A.F.: A high-resolution atlas and statistical model of the human heart from multislice ct. IEEE Transactions on Medical Imaging 32(1), 28-44 (2013)

18. Huysmans, T., Sijbers, J., Verdonk, B.: Automatic construction of correspondences for tubular surfaces. IEEE transactions on pattern analysis and machine intelligence 32(4), 636-51 (2010)

19. Jilaihawi, H., Kashif, M., Fontana, G., Furugen, A., Shiota, T., Friede, G., Makhija, R., Doctor, N., Leon, M.B., Makkar, R.R.: Cross-sectional computed tomographic assessment improves accuracy of aortic annular sizing for transcatheter aortic valve replacement and reduces the incidence of paravalvular aortic regurgitation. Journal of the American College of Cardiology 59(14), 1275-1286 (2012)

20. Kodali, S.K., Williams, M.R., Smith, C.R., Svensson, L.G., Webb, J.G., Makkar, R.R., Fontana, G.P., Dewey, T.M., Thourani, V.H., Pichard, A.D., Fischbein, M., Szeto, W.Y., Lim, S., Greason, K.L., Teirstein, P.S., Malaisrie, S.C., Douglas, P.S., Hahn, R.T., Whisenant, B., Zajarias, A., Wang, D., Akin, J.J., Anderson, W.N., Leon, M.B.: Two-year outcomes after transcatheter or surgical aorticvalve replacement. The New England journal of medicine 366(18), 1686-95 (2012)

21. Lacko, D., Huysmans, T., Parizel, P.M., De Bruyne, G., Verwulgen, S., Van Hulle, M.M., Sijbers, J.: Evaluation of an anthropometric shape model of the human scalp. Applied Ergonomics 48, 70-85 (2015)

22. Lekadir, K., Albà, X., Pereañez, M., Frangi, A.F.: Statistical Shape Modeling Using Partial Least Squares: Application to the Assessment of Myocardial Infarction, pp. 130-139. Springer International Publishing (2016)

23. Lorenz, C., von Berg, J.: A comprehensive shape model of the heart. Medical image analysis 10(4), 657-70 (2006)

24. Ltjnen, J., Kivist, S., Koikkalainen, J., Smutek, D., Lauerma, K.: Statistical shape model of atria, ventricles and epicardium from short- and long-axis $\{M R\}$ images. Medical Image Analysis 8(3), 371 - 386 (2004). Medical Image Computing and Computer-Assisted Intervention - \{MICCAI\} 2003 
25. Magnenat-thalmann, N., Seo, H., Cordier, F.: Automatic Modeling of Virtual Humans and Body Clothing. Journal of computer Science \& Technology 19(5), 575-584 (2004)

26. Paulsen, R.R., Larsen, R., Nielsen, C., Laugesen, S., Ersbøll, B.: Building and testing a statistical shape model of the human ear canal. Medical Image Computing and Computer-Assisted Intervention 2489, 373-380 (2002)

27. Pedregosa, F., Varoquaux, G., Gramfort, A., Michel, V., Thirion, B., Grisel, O., Blondel, M., Prettenhofer, P., Weiss, R., Dubourg, V., Vanderplas, J., Passos, A., Cournapeau, D., Brucher, M., Perrot, M., Duchesnay, É.: Scikit-learn: Machine Learning in Python. journal of Machine Learning Research 12, 2825-2830 (2011)

28. Pinto, C., Çimen, S., Gooya, A., Lekadir, K., Frangi, A.F.: Joint Clustering and Component Analysis of Spatio-Temporal Shape Patterns in Myocardial Infarction, pp. 171-179. Springer International Publishing (2016)

29. Ren, Y., Wang, L., Gao, Y., Tang, Z., Chen, K.C., Li, J., Shen, S.G.F., Yan, J., Lee, P.K.M., Chow, B., Xia, J.J., Shen, D.: Estimating anatomically-correct reference model for craniomaxillofacial deformity via sparse representation. Lecture Notes in Computer Science (including subseries Lecture Notes in Artificial Intelligence and Lecture Notes in Bioinformatics) 8674 LNCS(PART 2), 73-80 (2014)

30. Schultz, C.J., Moelker, A., Piazza, N., Tzikas, A., Otten, A., Nuis, R.J., Neefjes, L.A., Van Geuns, R.J., De Feyter, P., Krestin, G., Serruys, P.W., De Jaegere, P.P.T.: Three dimensional evaluation of the aortic annulus using multislice computer tomography: Are manufacturer's guidelines for sizing for percutaneous aortic valve replacement helpful? European Heart Journal 31(7), 849-856 (2010)

31. Sellers, R., Levy, M., Amplatz, K., Lillehei, C.W.: Left retrograde cardioangiography in acquired cardiac disease: Technique, indications and interpretations in 700 Cases. American Journal of Cardiology 14(October), 437-447 (1964)

32. Styner, M.a., Rajamani, K.T., Nolte, L.P., Zsemlye, G., Székely, G., Taylor, C.J., Davies, R.H.: Evaluation of 3D Correspondence Methods for Model Building. Proc. Information Processing in Medical Imaging 18, 63-75 (2003)

33. Thorstensen, N., tyngier, P., Sgonne, F., Keriven, R.: Diffusion maps as a framework for shape modeling. Computer Vision and Image Understanding 115(4), 520 - 530 (2011)

34. Thourani, V.H., Ailawadi, G., Szeto, W.Y., Dewey, T.M., Guyton, R.a., Mack, M.J., Kron, I.L., Kilgo, P., Bavaria, J.E.: Outcomes of surgical aortic valve replacement in high-risk patients: a multiinstitutional study. The Annals of thoracic surgery 91(1), 49-56 (2011)

35. Wang, Y., Yuan, L., Shi, J., Greve, A., Ye, J., Toga, A.W., Reiss, A.L., Thompson, P.M.: Applying tensor-based morphometry to parametric surfaces can improve MRI-based disease diagnosis. NeuroImage 74, 209-230 (2013)

36. Young, A.A., Frangi, A.F.: Computational cardiac atlases : from patient to population and back. Experimental Physiology 94(5), 578-596 (2009)

37. Zachow, S., Lamecker, H., Elsholtz, B., Stiller, M.: Reconstruction of mandibular dysplasia using a statistical 3D shape model. International Congress Series 1281, 1238-1243 (2005)

38. Zhao, F., Zhang, H., Wahle, A., Thomas, M.T., Stolpen, A.H., Scholz, T.D., Sonka, M.: Congenital aortic disease : 4D magnetic resonance segmentation and quantitative analysis. Medical Image Analysis 13(3), 483-493 (2009)

\section{Author's biographies}

Bart Bosmans obtained his MSc degree in biomedical engineering at the University of Leuven in 2011. His master thesis concerned modelling of mechanotransduction signaling in skeletal cells. He joined Materialise N.V. in 2011 as a research engineer and started a PhD in collaboration with the University of Leuven and the University of Antwerp in 2012. He held an IWT (Institute for the Promotion of Innovation through Science and Technology in Flanders) Baekeland mandate (2012-2016), his doctoral research focused on device implantation planning in soft tissue. Currently, he is a research engineer at at Materialise N.V. he focusses on research in image processing.

Toon Huysmans received the Master of Science degree in Computer Science in 2003. His graduation research concerned diamond cut optimization and was carried 
out at the Vision Lab of the University of Antwerp in close collaboration with the companies DiamCad and DiamScan. In 2003, he joined the Vision Lab as a PhD student where he enjoyed an IWT (Institute for the Promotion of Innovation through Science and Technology in Flanders) scholarship. His doctoral research focused on parameterization and correspondence construction for tubular surfaces and related applications. Currently, he is with the iMinds-Vision Lab as research manager, focusing on grant writing, guiding $\mathrm{PhD}$ students, and networking with industry. And as a post-doctoral researcher he does research on statistical shape modeling in ergonomics and orthopedics.

Patricia Lopes obtained her MSc degree in Biomedical Engineering at the University of Minho, Portugal, in 2007. Her master thesis, aimed at the selective laser melting of a personalized triflange acetabular cup implant, was carried out at the KULeuven and the University of Minho. In 2008, she joined Materialise N.V. and now holds an IWT (Institute for the Promotion of Innovation through Science and Technology in Flanders) Baekeland mandate for a doctoral project focused on the 4D characterization of the mitral valve apparatus for transcatheter device design.

Eva Verhoelst obtained her MSc (2005) and PhD (2011) degree in bioscience engineering at the University of Leuven. She received an IWT (Institute for the Promotion of Innovation through Science and Technology in Flanders) grant for her PhD research entitled: Structural Analysis of the Angiogenesis in the Chicken Chorioallantoic Membrane. Currently she is a research project manager at Materialise N.V.

Tim Dezutter obtained his MSc degree in biomedical engineering at the University Ghent in 2012. His graduation thesis concerned virtual and experimental transcatheter aortic valve implantation bench testing. He joined FEops N.V. as research and development engineer in 2012, holds an IWT (Institute for the Promotion of Innovation through Science and Technology in Flanders) Baekeland mandate and does research into fatigue testing of cardiovascular devices. He is currently working towards a $\mathrm{PhD}$ degree in collaboration with the University Ghent.

Peter de Jaegere received his MD degree from the University Ghent, Belgium in 1983 and followed training in Cardiology at the Free University Brussels, Belgium and the University Hospital Rotterdam, The Netherlands (1983-1990). From 1990-1996, he was staff member and clinical head of the Department of Cardiac Catheterization \& Interventional Cardiology in Rotterdam. He was in charge of the introduction and evaluation of intracoronary stenting, which lead to his $\mathrm{PhD}$ thesis Clinical and Angiographic Evaluation of Intracoronary Stenting in Humans (1993) and, the publication of the Benestent Study (randomized comparison balloon angioplasty vs. intracoronary stenting) in the New England Journal of Medicine in 1994. From 1996-2004 he was in charge of the clinical research program at the Department Cardiac Catheterisation \& Interventional Cardiology at the University Hospital Utrecht. In 2004 he returned to Rotterdam where he set up the Catheter Based Valve program.

Jan Sijbers graduated in Physics in 1993. In 1998, he received a PhD in Physics from the University of Antwerp, entitled: Signal and Noise Estimation from Magnetic Resonance Images". He was an FWO (Fund for Scientific ResearchFlanders) Postdoc at the University of Antwerp and the Delft University of Technology from 20022008. In 2005, he became a lecturer and in 2010 senior lecturer. In 2014, he was 
appointed full professor at the University of Antwerp. He is Associated Editor of IEEE Transactions on Image Processing as well as of IEEE Transactions on Medical Imaging. Furthermore, he is in the editorial board of Physica Medica. Jan Sijbers is the head of iMinds-Vision Lab and co-founder of IcoMetrix.

Jos Vander Sloten obtained his MSc and PhD in mechanical engineering from the University of Leuven (KU Leuven). From 1985 to 1999 he held positions as research assistant and post-doctoral researcher of the Fund for Scientific Research Flanders, specialising in musculoskeletal biomechanics. Currently he is full professor and chair of the Division of Biomedical engineering at K.U.Leuven. He also chairs the Leuven Medical Technology Centre (L-MTC). His teaching assignments are mechanics, problem solving and engineering design, and computer integrated surgery systems. His research interests are computer applications in musculoskeletal biomechanics and computer integrated surgery. He is member of the council of the Belgian Society for Medical and Biological Engineering and Computing, and a former council member of the European Society of Biomechanics. In the European Alliance for Medical and Biological Engineering and Science (EAMBES) he served as secretary-general (2003-2004), president-elect (2005), president (2006) and vicepresident (2008-2009).

Johan Bosmans holds both an academic and clinical post at the University Hospital of Antwerp. He is a lecturing professor, is experienced in clinical research and is an internationally renowned cardiologist. Prof. Bosmans is a member of the medical ethics committee of the University Hospital, a board member of the Belgian Society of Cardiology and a member of the Belgian Interdisciplinary Working Group on Acute Cardiology. His main research interests are related to TAVI and predicting outcome after transcatheter aortic valve implantation. 